Chapter 9

\title{
Radiation and Chemotherapy in Oral and Maxillofacial Surgery
}

\author{
Orett E. Ogle and Levon Nikoyan \\ Additional information is available at the end of the chapter \\ http://dx.doi.org/10.5772/53402
}

\section{Introduction}

Oral cavity cancer is the sixth most prevalent cancer worldwide(1]and comprise about $85 \%$ of all head and neck cancers. Regions with a high incidence of oral cancer $(>6.9 / 100,000]$ are : North America, Brazil, Europe, South Africa, the Indian Subcontinent, and Australia Areas with low incidence $(<3.2 / 100.000]$ are Central America, Chile, West Africa, Middle East and China. [2]The higher incidence of oral cancer in high income countries, and increasingly in middle-income countries, is thought to be due to tobacco usage, unhealthy diets, alcohol consumption, inactive lifestyles and infection. The use of tobacco, including smokeless tobacco, and excessive consumption of alcohol are regarded as the major risk factors for oral cancer. [1]Although oral cancer originates from different types of tissues that are present in the mouth, around $85-90 \%$ are squamous cell carcinomas originating in the oral epithelium. The treatment of oral cancers is ideally a multidisciplinary approach involving the efforts of surgeons, radiation oncologists, chemotherapy oncologists, dental practitioners, nutritionists, and rehabilitation and restorative specialists. Curative treatment modalities are usually surgery and radiation, with chemotherapy added to sensitize the malignant cells to radiation, to decrease the possibility of metastasis, or as curative treatment for those patients who have confirmed distant metastasis. The factors that influence the choice of treatment modality are related to the tumor and the desires of the patient. Primary site, size of the tumor, lymph node involvement and the presence or absence of distant metastasis are factors which will affect a particular treatment option. Surgery is the most common treatment for mouth cancer, while oropharyngial cancer is usually treated with radiation, with or without chemotherapy. Most oncologist consider radiotherapy or chemoradiotherapy (CRT) as first-line therapy in oropharynx cancer due to the equivalent response rates compared with surgery. Salivary gland tumors are commonly treated with surgery initially. In general, Stage I and Stage II oral cancers may be treated successfully with either surgery or radiation therapy. Advanced Stage III and Stage IV cancers are typically treated by surgical resection followed by radiotherapy (RT) or CRT 


\section{Radiotherapy}

Technological improvements in machines and techniques used for radiation therapy has given radiotherapy an advantage as the primary modality for treating oral cancer by having less patient morbidity and being well tolerated. Radiation therapy for oral cancer will be delivered either by external beam therapy (EBT) or by intensity-modulated radiation therapy (IMRT). EBT is administered with machines called linear accelerators, which produce high-energy external radiation beams. This beam or beams of radiation penetrates the tumor delivering tumorcidal doses. The newer linear accelerators have enabled radiation oncologists to significantly reduce side effects while improving the capacity to deliver radiation to the cancer with better cure rates. IMRT is an advanced mode of high-precision radiation therapy that utilizes computer-controlled x-ray accelerators to deliver controlled radiation doses to a malignant tumor or specific areas within the tumor. IMRT allows the precise delivery of high doses of radiation to the tumor while minimizing damage to adjacent tissues due to the sharp dose falloff gradient between the gross tumor and the surrounding normal tissue. IMRT can conform to the irregular shape of a tumor, delivering higher doses directly to the tumor cells with the added potential of also destroying more radioresistant cells. Numerous data have suggested that IMRT provides locoregional control in $90 \%$ of cases and is well tolerated by patients. (3]

\subsection{Radiation protocols}

A large number of radiotherapy techniques and protocols exist for the treatment of head and neck cancers. Deciding which technique to use is generally a complex one as it depends on the size, location and cellular components of the tumor.[4] As mentioned earlier, treatment modalities can be broadly classified into external beam therapy and intensity-modulated radiation therapy with few other techniques that have recently gained popularity in treatment of the head and neck cancer (Table 1). [5]

\begin{tabular}{|c|c|c|}
\hline \multicolumn{3}{|c|}{ Radiation Therapy } \\
\hline Technique & Description & Cancers Used \\
\hline Brachytherapy & Radiation source is placed inside or near the tumor. & $\begin{array}{l}\text { Many, including head } \\
\text { and neck }\end{array}$ \\
\hline Intensity-modulated Radiation Therapy & $\begin{array}{l}\text { High-precision radiotherapy that uses accelerators to deliver precise } \\
\text { radiation doses to the tumor }\end{array}$ & $\begin{array}{l}\text { Head and Neck, } \\
\text { Prostate, Lung, Brain }\end{array}$ \\
\hline Gamma Knife & $\begin{array}{c}\text { Radiosurgery, selective ionization of tissue, by means of high-energy } \\
\text { beams of radiation. Production of ions and free radicals which are } \\
\text { deleterious to the target cells. }\end{array}$ & Brain, prostate \\
\hline Total Body Irradiation & Total Body Irradiation to suppress host's immune response. & $\begin{array}{l}\text { Leukemia, Myelomas, } \\
\text { Bone Marrow } \\
\text { transplant }\end{array}$ \\
\hline Charged Particle Radiotherapy & $\begin{array}{c}\text { External beam radiation that uses protons and heavy particles as } \\
\text { opposed to photons to deliver radiation. }\end{array}$ & $\begin{array}{l}\text { Nasopharyngeal } \\
\text { tumors. Sarcomas, } \\
\text { Melanomas }\end{array}$ \\
\hline Neutron Radiotherapy & Use of high energy, fast neutrons to delivery energy. & $\begin{array}{l}\text { Salivary gland, } \\
\text { prostate }\end{array}$ \\
\hline Targeted Radionuclide Therapy & $\begin{array}{c}\text { Use of Antibodies as carriers of radio nucleotides. } \\
\text { Radioimmunotherapy }\end{array}$ & $\begin{array}{l}\text { Many types based } \\
\text { on antibodies }\end{array}$ \\
\hline
\end{tabular}

Table 1. List of commonly utilized radiation techniques. Modified from Clinical Radiation Oncology. 3rd ed. Leonard L. Gunderson JET, editor. Philadelphia: Saunders; 2012 
External Beam, IMRT and brachytherapy are most commonly used in the head and neck region. Table 2 presents the list of most commonly occurring head a neck tumors and their general treatment rational and radiation sensitivity. [6]

\begin{tabular}{|c|c|c|c|}
\hline Tumor & Radiotherapy & Chemotherapy & Surgery \\
\hline $\begin{array}{l}\text { Squamous Cell } \\
\text { Carcinoma }\end{array}$ & + & + & + \\
\hline Basal Cell Carcinoma & + & & + \\
\hline Kaposi's Sarcoma & + & + & + \\
\hline Melanoma & + & + & + \\
\hline $\begin{array}{l}\text { Leiomyoma and } \\
\text { Leiomyosarcoma }\end{array}$ & & & + \\
\hline $\begin{array}{l}\text { Rhabdomyoma and } \\
\text { Rhabdomyosarcoma }\end{array}$ & + & + & + \\
\hline $\begin{array}{l}\text { Mucoepidermoid } \\
\text { Carcinoma }\end{array}$ & + & & + \\
\hline $\begin{array}{l}\text { Polymorphous Low- } \\
\text { Grade Adenocarcinoma }\end{array}$ & + & & + \\
\hline $\begin{array}{l}\text { Adenoid Cystic } \\
\text { Carcinoma }\end{array}$ & + & & + \\
\hline Actinic Cell Carcinoma & + & & + \\
\hline Clear Cell Carcinoma & & & + \\
\hline Hodgkin's Lymphoma & + & + & \\
\hline $\begin{array}{l}\text { Non Hodgkin's } \\
\text { Lymphoma }\end{array}$ & & + & \\
\hline Multiple Myeloma & & + & \\
\hline $\begin{array}{c}\text { Solitary Plasmocytoma } \\
\text { of Bone }\end{array}$ & + & & + \\
\hline Leukemia & & + & \\
\hline Osteosarcoma & + & + & + \\
\hline Chondrosarcoma & & & + \\
\hline Ewing's Sarcoma & + & + & + \\
\hline Burkitt's Lymphoma & & + & \\
\hline Granular Cell Tumor & & & + \\
\hline Schwannoma & & & + \\
\hline Neurofibroma & & & + \\
\hline
\end{tabular}

Table 2. Treatment of selected tumors. Modified from Ang KK. Advances in the Treatment of Head and Neck Cancer. In: James D. Cox KKA, editor. Radiation Oncology, Treatment, Technique Rationale. 9th ed. Philadelphia, PA: Mosby, Elsevier; 2010. p. 161-353

External beam radiotherapy depends on photons, moving packets of energy that deliver radiation to the tissues. When photons interact with matter, electrons are displaced from their orbits around the nucleus of the atoms in the irradiated tissue. The atom is left with a positive charge, and thus becomes an ion/'free radical', (hence the term "ionizing radiation").[7] The process continues with ionized particles transferring energy and setting more particles in motion. As the particles travel through the matter, however they continuously loose energy with the maximum loss occurring just before they come to rest. (Bragg's peak)[8] The depth in the tissue that the Bragg's peak occurs is dependent on the source of photons, and this is selected by the oncologist when determining what X-ray energy to prescribe. Radiation in the external beam therapy 
is generated by linear accelerators. These are complex units that accelerate electrons by providing alternating microwave fields and are capable of focusing energy to accommodate target size. [9] These accelerators are capable of producing a large range of $X$-ray energy from $50 \mathrm{kV}$ to $20 \mathrm{MV}$. For head neck cancers however the most useful range lies in $50-150 \mathrm{kV}$, the so-called superficial X-rays. This range is useful for most skin and mucosal cancers. At times when a larger radiation is required, orthovoltage $X$-rays can be utilized. This is radiation in the range of 200-300 $\mathrm{kV}$ and can penetrate tumors as deep as $3 \mathrm{~cm}$. Intensity modulated radiotherapy (IMRT) is part of conformal therapy which as an advanced radiotherapy modality that relies on computerized tomography to calculate and recreate tumor's exact volume. Intensity modulation refers to the X-ray's beams variable strength to deliver exact radiation to the tumor proving maximum sparing to adjacent tissues. The overall process is outlined in Figure 1.

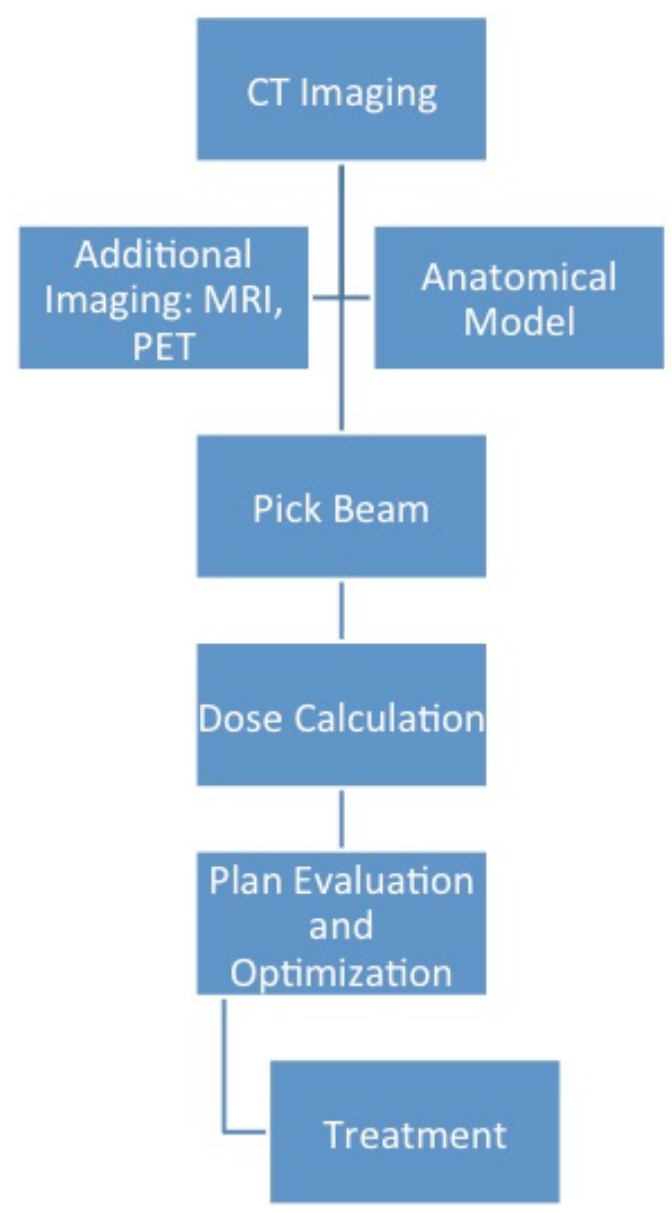

Figure 1. Intensity modulated radiotherapy treatment planning. Modified from Clinical Radiation Oncology. 3rd ed. Leonard L. Gunderson JET, editor. Philadelphia: Saunders; 2012 
The treatment planning begins after obtaining an appropriate CT scans of the tumor area as well any other studies that will facilitate creation of a complete volumetric analysis (including soft tissue) as well as functional imaging of the tumor area. Three-dimensional model of the tumor area is then created inside a wide variety of specialized planning software. If needed 4$\mathrm{D}$ imaging can also be utilized to accommodate motion (i.e. when lungs/ cardiac tissue is examined). Once the model is completed gross target volume is defined from the CT image and the radiation oncologist defines the clinical target volume. This is the volume of the tumor plus any additional area that should be treated.

\subsection{Dental preparation of the patient for radiation}

The status of the dentition has a significant effect on post-treatment quality of life among patients with head and neck cancer that will undergo radiation. A dentition in poor repair will increase the risk of post-radiation complications, particularly dentoalveolar infections that could lead to osteoradionecrosis. All patients who will be treated with RT for oral/head and neck cancer should undergo a comprehensive dental evaluation prior to treatment.Carious teeth, teeth with deep restorations or in poor periodontal health, along with partial bony impacted third molars should be extracted prior to RT if in an area that is expected to receive a dose of at least $50 \mathrm{~Gy}$. Teeth that are out of the radiation treatment field, but have a hopeless prognosis or is symptomatic should also be extracted. Extraction of healthy teeth does not appear to prevent the development of osteoradionecrosis.[10]All indicated extractions should be completed prior to RT and primary closure over the extraction sites is preferred if possible. An adequate alveoloplasty should be performed to eliminated the possibility of bone edges ulcerating the mucosa as well as to make the mandible/maxilla ready for dentures. Ideally, all extractions should be completed approximately two weeks before the commencement of RT to permit proper healing. If the extracted teeth are outside of the treatment areas, however, radiation may be started sooner. The oral surgeon should attempt to do all the extractions within the portals of radiation at one sitting so as not to delay the cancer treatment. Postponing needed extractions of teeth that will be within the treatment area until after radiation is associated with an increased risk of non-healing and osteoradionecrosis.

\subsection{Management of radiation associated problems}

Radiotherapy in the upper aerodigestive tract can cause a wide spectrum of toxicities. The most basic toxicities are the impairment in the ability to breathe, communicate, and maintain an adequate oral intake. Oral intake is compromised by swallowing problems (dysphagia and odynophagia), poor taste (dysgeusia),trismus, xerostomia, and mucositis. In addition, there may be added dental complications from the effects of radiation dose to the mandible/maxilla and salivary glands. Acute toxicity is defined as events that occur during radiation therapy or within 90 days after the commencement of treatment and are largely unavoidable but transient. Late toxicity, can be minimized but is generally long-lasting and in some instances permanent. 


\subsection{Salivary gland damage and xerostomia}

Decreased saliva production becomes evident within one to two weeks after the initiation of RT, and permanent reduction can be noted with cumulative radiation doses as low as 10 to 15 Gy to the parotid gland. [11] Doses greater than 24 to 26 Gy will cause permanent damage to the parotid glands. This can decrease the production of saliva from $40-80 \%$. During and immediately after treatment, patients should be instructed to drink adequate fluids and to rinse and gargle with either a dilute solution of 25 percent hydrogen peroxide and 75 percent water or a weak solution of salt and baking soda (one-half teaspoon of salt and one teaspoon of baking soda added to one quart of water) several times daily. This regimen can loosen thick, tenacious oral secretions, and alleviate pain due to mild mucositis.[12] Amifostine is a drug that can reduce the incidence of xerostomia in patients undergoing radiotherapy for head and neck cancer. Although it is the only pharmacologic agent with established efficacy in the prevention of xerostomia, its role in patient management is uncertain and the use of amifostine is not standard. For patients that have lasting post-radiation xerostomia, pilocarpine may be used to stimulate saliva production from residual salivary gland tissue. However, pilocarpine is not recommended to prevent xerostomia in patients receiving RT for head and neck cancer.

\subsection{Mucositis}

From the second or third weeks onwards, almost all patients undergoing head and neck cancer RT will experience mucositis. Radiation-induced loss of stem cells in the basal layer interferes with the replacement of cells in the superficial mucosal layers when they are lost through normal physiologic sloughing. The subsequent denuding of the epithelium results in mucositis, which is painful and will interfere with oral intake and nutrition. Mucositis is managed symptomatically. Good oral hygiene is imperative. Dietary modification will be necessary, and topical agents for superinfections and pain may be required. The patient should avoid acidic or spicy foods, sharp foods (eg, chips), caffeine, alcoholic beverages and alcohol-containing mouthwashes. Secondary bacterial, fungal (oral candidiasis), and viral (herpes) infections should be treated with appropriate agents. Localized mouth pain can be treated with topical anesthetics ( example $2 \%$ viscous lidocaine). This may be combined with an antacid suspension (Mylana,Maalox, Gelusil) and/or diphenhydramine (for local drying effect). Dexamethasone solution (an anti-inflammatory), tetracycline suspension (antibiotic) or nystatin (antifungal) may also be added to the mixture.

\subsection{Dysgeusia}

An abnormal or impaired sense of taste ( the sense of taste may also be affected by impaired olfaction). An altered sense of taste and/or smell may contribute to nutritional difficulties and weight loss; $67 \%$ of patients treated by RT have dysgeusia. There is no successful treatment for this problem and dietary counseling should be instituted to counteract a lack of appetite. 


\subsection{Orofacial pain}

Long-acting opiates (oxycontin, levorphanol, oxymorphone) should be used as needed during the treatment period. For patients who cannot swallow oral medication, transdermal fentanyl may provide good pain relief. Short-acting opiates (morphine, codeine, oxycodone) should be used for breakthrough pain.

\subsection{Trismus}

Limited jaw opening during therapy is typically secondary to pain. For that reason, passive motion devices are generally not used during radiotherapy. By contrast, passive motion devices (TheraBite, E-Z Flex) can generally be instituted early in the postoperative period. Adequate pain control will be necessary.

\section{Osteoradionecrosis}

One of the most serious complications of radiation therapy is postradiation treatment necrosisosteoradionecrosis. This is generally a delayed onset disease that usually takes significant radiation to develop and manifests itself after the irradiated area is subjected to dental surgery, trauma and ongoing periodontal infection. [13-15] Although osteoradionecrosis is closely associated with above mentioned factors, spontaneous appearance of the disease is not unusual with documented incidence in the literature.[16] The incidence of osteoradionecrosis is variable with $2.6 \%$ to $22 \%$ for the mandible with significantly lower incidence in the maxilla. $[17,18]$ The precise mechanism of injury is still unknown but the progression the disease begins with a slow change in the matrix of bone after irradiation. The initial changes result from injury to the remodeling system, i.e. the osteocytes, osteoblasts, and osteoclasts. Osteoblasts tend to be more radiosensitive than osteoclasts, leading to increase in the initial destruction of bone. $[19,20]$ The later changes result from alterations of the vascular system itself; causing fundamental damage to bone architecture. Radiation injury to the fine vasculature of the bone first leads to hyperemia, followed by endarteritis, thrombosis, and progressive occlusion and obliteration of the small vessels. This results in a further reduction of the number of cells and progressive fibrosis within the bone. With time, the marrow exhibits marked acellularity, hypo- or avascularity, and significant fatty degeneration and fibrosis. The endosteum atrophies with significant loss of active osteoblasts and osteoclasts. The periosteum demonstrates remodeling with significant overall loss of blood supply.[21] Although in the past osteoradionecrosis was mainly considered an infectious entity[22, 23], it is currently accepted to be a problem of wound healing with infecting organisms being mostly contaminants. [16] According to this model the hypoxic, hypovascular, hypocellular tissues have reduced ability to replace normal cellular and collagen loss, which eventually results in tissue breakdown. The weakened tissues have reduced ability to heal relevant wounds, since the metabolic demands exceed the vascular supply. The signs and symptoms of osteoradionecrosis will usually begin sometime after the radiation therapy. First as an exposed, non-healing area of the bone that progressively enlarges and becomes painful. The area can show evidence of secondary 
infection with progression to sequestrate formation, cutaneous fistulae and even pathologic fractures. $[18,24]$ The diagnosis of osteoradionecrosis is established on a combination of clinical features and radiological features. Plain dental radiographs show decreased bone density. Computed tomography scans show bone abnormalities, such as focal lytic areas and cortical breaks. MRI and as well as bone scans can also be helpful in diagnosis. [25, 26]

Prevention of osteoradionecrosis is centered on patient education and reduction of risks factors. All carious and non-restorable teeth should be extracted prior to the beginning of the radiation therapy. Periodontal concerns should be addressed and any teeth with questionable prognosis should be strongly considered for extractions. If the oral hygiene is controlled and considered dental surgery is completed 14-21 days prior to beginning of the radiotherapy the risk of developing osteoradionecrosis becomes insignificant. [27]Extraction of teeth during and after radiation therapy posses a significant risks for osteoradionecrosis. [28, 29] If extractions are required in post-radiation therapy then atraumatic surgery is indicated with tension free primary closure. Antibiotic coverage is also advised with either penicillin or clindamycin. [18, 30]Hyperbarric oxygen therapy should be strongly considered for prevention and treatment of osteoradionecrosis (see next section).

The mainstay of treatment of osteoradionecrosis remains antibiotic treatment with limited curettage, debridement and removal ofsequestrae. More extensive surgical therapies are indicated for refractory lesions [31]. The first step is debridement of all bone that is no longer vascularized; as this dead bone, if not removed, will continue to promote further bacterial growth. Further and more invasive surgical techniques include extensive sequestrectomy combined with marginal or complete resection of affected parts of the mandible (and stabilization of the continuity defect). Hyperbaric oxygen therapy should be strongly considered as it has been shown to improve healing. [32]

\section{Hyperbaric oxygen}

Hyperbaric oxygen is defined as administration of $100 \%$ oxygen under pressure that is significantly higher than the ambient pressure. For patients receiving hyperbaric oxygen it is possible to administer nearly fifteen times more oxygen. [33] The use of hyperbaric oxygen (HBO) therapy in osteoradionecrosis is based on the principle that oxygen stimulates collagen synthesis, matrix deposition, angiogenesis, epithelialization, and the eradication of bacteria. [34]The immediate effects of breathing high concentrations of oxygen in increased pressure causes an increase in the tissue's internal oxygen pressure, leading to vasoconstriction, enhanced oxygen delivery, edema reduction, phagocytosis activation, and an antiinflammatory effect. The long-term effects are neovascularization, osteogenesis, and a stimulation of collagen production by fibroblasts, all promote wound healing. Generally, two types of chambers exist: monoplace and multiplace. The monoplace chamber is a HBO chamber that is suited for only one patient with no direct access to the patient while he or she is receiving the therapy. Multiplace, ICU compatible chambers on the other hand are able to accommodate multiple patients and even nursing staff. Although the exact mechanism of HBO therapy is 
not understood, what is known is that $\mathrm{HBO}$ appears to reverse some of the deleterious effects of radiation on bone. Several studies that focused on HBO's effects on osteoblast proliferation have concluded that $\mathrm{HBO}$ has an effect in increasing osteoblasts differentiation into osteogenic phenotype but not necessarily overall increase in cellularity of the bone.[35]. Use of HBO in treatment of osteoradionecrosis was discussed as early as 1983, when Marx proposed staging based on disease progression and response. [16] This was later addressed by Kagan and Schwartz when they proposed a three-stage system where the disease is classified based on clinical and radiologic findings and treatment is determined based on the stage, similar to the approach for malignancies of the head and neck.[36] Figure 2 summarizes treatment proposed by Marx that is dependent on disease response to HBO therapy.

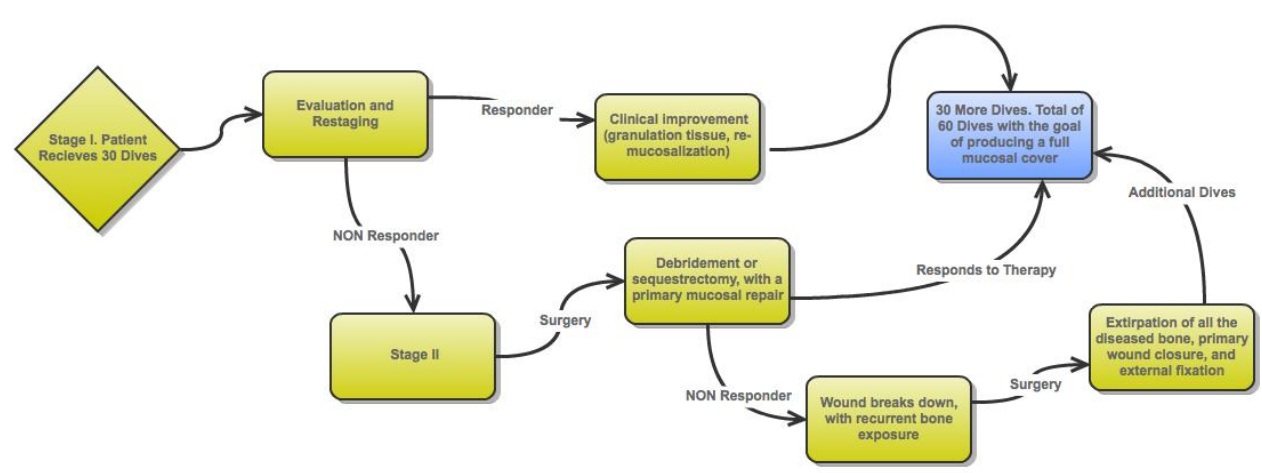

Figure 2. HBO Treatment protocol based on response to HBO, modified from Marx RE. A new concept in the treatment of osteoradionecrosis. J Oral Maxillofac Surg. 1983 Jun;41[6]:351-7.

Current treatment protocols vary considerably but they include utilization of panoramic imaging and CT findings in conjunction with clinical findings to determine if a patient has early, intermediate, or advanced stage disease. [37]Table 3 summarizes currently accepted treatment protocols.

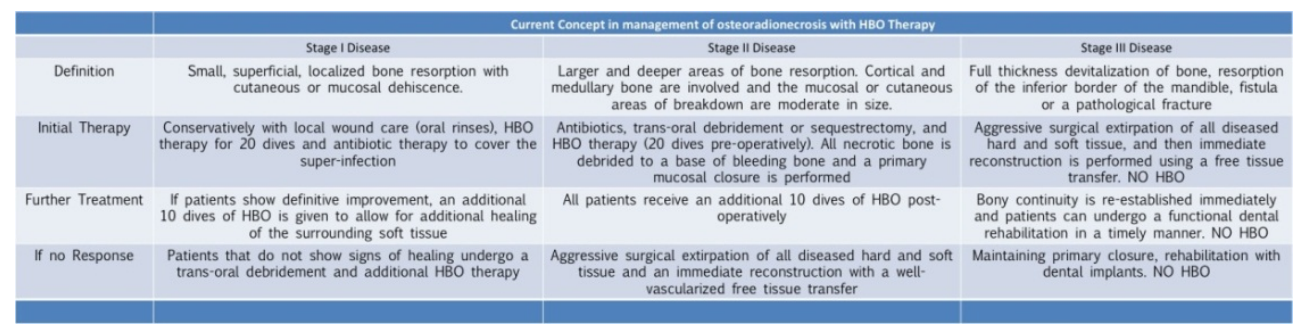

Table 3. Summary of Current HBO use in Osteoradionecrosis. Modified from Jacobson AS, Buchbinder D, Hu K, Urken ML. Paradigm shifts in the management of osteoradionecrosis of the mandible. Oral Oncol. 2010 Nov;46[11]:795-801 


\section{Surgery in the post-irradiated patient}

Dental extractions or minor oral surgery in patients who have undergone radiation therapy for cancer in the head and neck carry the risk of one of the most serious and devastating complications of head and neck radiotherapy, that of osteoradionecrosis (ORN). Elective oral surgery on irradiated bone should therefore be avoided. The risk of ORN does not decrease with time. When contemplating exodontia or minor oral surgery in the irradiated patient, special consideration should be given to issues such as radiotherapy history, surgical assessment, surgical procedure and the role of antibiotics and hyperbaric oxygen.

The actual field of radiation should be noted as extractions performed outside the area of radiation do not constitute a risk factor to the development of ORN. Ionizing radiation causes irreversible cellular and vascular damage resulting in hypoxic, hypocellular and hypovascular tissue. This fact greatly affects the reparation process and there is a consensus that extractions in irradiated fields must be executed with as little trauma as possible. Sectioning multi-rooted teeth, gentle elevation of roots, alveolectomy with careful bone trimming, conservative flaps, primary closure without tension and removal of few teeth per session minimize postoperative complications and are associated with lower ORN rates.[38] Prophylactic antibiotics should be used as adjuvant therapy and the antibiotics continued for $10-14$ days post-extraction. There is no consensus about the employment of antibiotics to prevent ORN however, and some authors have expressed the opinion that an antibiotic as a sole agent is not sufficient to reducing the risk of ORN. Once dental extractions become unavoidable after radiotherapy, it should be done with adjuvant therapies - hyperbaric oxygen (HBO) with or without antibiotics- and rigorous follow-up after the surgical procedures.

The subject of placing dental implants in irradiated bone is not clear. Some papers have shown that implants can be successfully oseointegrated if $\mathrm{HBO}$ is used as an adjuvant therapy. [39] On the other hand, Franzen et al reported a $95 \%$ (19/20 implants) successful osseointegration with Brånemark implants placed in irradiated mandibles with stability of the implants after 3 to 6 years of observation. Their oral surgical procedures were carried out without adjunct hyperbaric oxygen therapy, and their successful results demonstrates that adjunctive measures are not always necessary in the oral rehabilitation after radiotherapy.

Soft tissue radionecrosis results from damage done to non-osseous tissues by ionizing radiation during the course of radiotherapy. Once the patient is exposed to the radiation beam, the soft tissue will begin to manifest ischemic changes. Ischemic tissue may survive without adequate blood supply for a long period of time, until a traumatic or infectious incident triggers the events leading to extensive tissue death - soft tissue radionecrosis. Surgeons attempting maxillofacial surgery in or adjacent to the radiated area will confront numerous complications. Oozing of blood during the procedure is common and difficult to control. Incisions made through irradiated tissue may not heal and the risk of infection is increased.

After the first post-irradiation year the most significant problems arising during this period result from chronic deterioration of the microvasculature with resulting hypoperfusion and tissue hypoxia. Such developments trigger an increasing tissue fibrosis, 
parenchymal degeneration, and lower resistance to micro-organisms and trauma. The situation can be optimized by bringing additional blood supply to the area via a vascularized flap or by using HBO. Treatment with hyperbaric oxygen therapy has remarkably changed the treatment of soft tissue necrosis disease. HBO allow tissues and vessels to be hyperoxygenated and promotes healing.

\section{Role of chemotherapy in head and neck cancer}

The use of chemotherapy in head and neck cancer has evolved greatly over the last three decades. While it was initially confined to patients with recurrent or metastatic disease, it is now frequently used as an initial curative component of combined modality therapy. When combined with radiation therapy,chemotherapy has been shown to enhance the effectiveness of the radiation making it more active against tumor cells. Chemotherapy by itself, however, has not changed the recurrence rate of oral squamous cell carcinoma but it has increased the rates of organ preservation and decreased the rates of distant metastasis when combined with radiotherapy. Chemotherapeutic agents also have a role in the palliative treatment of squamous cell cancer of the head and neck. To date, the agents found to be most effective for treating oral cancer include cisplatinum, carboplatinum, taxanes, 5-fluorouracil, methotrexate, and ifosphamide. These agents have been used alone or in combination in a variety or regimens. The agents vary in their single agent response rate and toxicity.

Outside of the head and neck, chemotherapy is used for cancer patients that are not curable by regional modalities (surgery and/or radiation) and is, at this time, the best adjuvant to local therapy in a wide range of human malignancies. Although some tumors are treated with a single medication, chemotherapy regimens most often involve the use of several antineoplastic drugs (combination therapy).

All chemotherapeutic agents act by interfering with cell division and are most active against rapidly dividing cells. Malignant tissues are made up of rapidly dividing cells characterized by rapid synthesis of DNA, and non-dividing cells with slower DNA synthesis. Most of the drugs used in chemotherapy work by affecting either enzymes or substrates acted upon by enzyme systems which relate to DNA synthesis or function. For treating cancer the majority of the agents exploit kinetic differences between normal and malignant cells by acting preferentially on the cells that are dividing at a faster rate. Consequently, malignant cells will be destroyed faster than normal cells at the tumor site. However, normal cells that have a high proliferative capacity rivaling malignant cells (bone marrow, gastrointestinal mucosa, oral mucosa, skin and hair follicles) will also be severely affected. The side effects of chemotherapeutic agents, therefore, include: myelosuppression (leukopenia, thrombocytopenia and anemia), nausea, vomiting, diarrhea, mucosal ulceration, dermatitides and alopecia.

Oral and maxillofacial surgeons will generally not be treating oral cancer patients with chemotherapy. They may, however, need to manage these patients for oral surgical procedures.

The surgical and anesthetic considerations of patients on cancer chemotherapy will be related primarily to an awareness of the multiplicity of noxious side effects presented by the various 
drugs. Preoperative evaluation will consist of a thorough history and physical exam, with focus on the clinical effects of the negative side effects which could increase morbidity and mortality. Routine laboratory test should include: $\mathrm{CBC}$, serum electrolytes and urinalysis. Depending on the drug, and other findings a LFT, chest X-ray, EKG and platelet function tests may be required.

\subsection{Chemotherapeutic drugs}

Chemotherapy drugs are classified according to how they work. The main types of chemotherapy drugs are described below along with their noxious effects.

\subsubsection{Alkylating agents}

Alkylating drugs undergo electrophilic chemical reactions that result in the formation of covalent links (alkylation) with DNA. The 7-nitrogen atom of guanine residues in DNA is particularly susceptible to formation of a covalent bond which results in a miscoding of DNA information or opening of the purine ring with damage to the DNA molecule. The alkylating agent can act on the DNA molecule at any stage of cell division.

Side effects: Bone marrow suppression is the most important- lymphocytopenia is usually present within 24 hours. Hemolytic anemia, alopecia, nausea and vomiting occurs commonly. Inhibition of plasma cholinesterase activity can cause prolonged skeletal muscle paralysis after administration of succinylcholine. Pneumonitis and pulmonary fibrosis may also occur.

Plant alkaloids: Referred to as "Vinca alkaloids" arrest cells in the metaphase of mitosis by binding to tubulin and thereby inhibiting microtubular function. Useful Vinca alkaloids derived from the periwinkle plant are Vinblastine and Vincristine. Paclitaxel is an extract of the bark of the Pacific yew. Despite their structural similarity, there is a not cross tolerance between them.

Side effects: Myelosuppression (leukopenia, thrombocytopenia and anemia) is the most common and appears 7 - 10 days after the start of therapy. Other commonly occuring side effects are: symmetric peripheral sensory-motor neuropathy, ataxia and transient depression. Autonomic neuropathy with orthostatic hypotension, bowel motility dysfunction, and cranial nerve involvement \{weakness of extraocular muscles and laryngeal nerve paralysis with hoarseness) are seen in $10 \%$ of patients. SIADH occurs with vincristine.

\subsubsection{Antimetabolites}

Antimetabolites act as fraudulent analogues of vital physiological substrates that inhibit the synthesis of DNA or its nucleotide building blocks. They include analogues of folic acid (methotrexate), pyrimidine (cytosine arabinoside) and purine (6-mercaptopurine). These drugs interact directly with specific enzymes, leading to inhibition of that enzyme and subsequent synthesis of an aberrant molecule that functions abnormally. These drugs are immunosuppressants. 
Note: Methotrexate is sometimes used for the treatment of some nonmalignant disorders: psoriasis and rheumatoid arthritis.

Side effects: Depends on the analogue that is used..

Methotrexate: GI (ulcerative stomatitis and diarrhea) and bone marrow (leukopenia and thrombocytopenia) side effects are most common. Hemorrhagic enteritis and death from intestinal perforation are other common side effects. Renal toxicity $(10 \%)$ and pulmonary toxicity $(8 \%)$ may also occur.

Cytosine arabinoside:Myelosuporession (leukopenia, thrombocytopenia and anemia) is the most common, GI disturbance, stomatitis and hepatic dysfunction also occurs less frequently.

Mercaptopurine: The principal side effect is a gradual development of bone marrow depression resulting in thrombocytopenia, granulocytopenia and anemia. Anorexia, nausea and vomiting are also common. Jaundice associated with bile stasis, and occasionally hepatic necrosis, occurs in $30 \%$ of patients.

\subsubsection{Antitumor antibiotics}

Antitumor antibiotics are natural products of certain soil fungi. Their effects are produced by the formation of relatively stable complexes with DNA, thereby inhibiting DNA synthesis, RNA synthesis, or both. Like antibiotics used for their antimicrobial activities these antitumor antibiotics all act differently. Some commonly used antibiotics are:

Acinomycin D (Dactinomycin): Binds to DNA in rapidly proliferating cells blocking RNA polymerase and thus the transcription of DNA.

Bleomycin: Water solubleglycopeptides that differ from one another in their terminal amine moiety (there are more than 200 congeners). They cause fragmentation of DNA..

\subsubsection{Enzymes}

L-asparaginase is an enzyme with useful chemotherapeutic effects. It depletes cells of the nonessential amino acid asparagine. Most human tissue have the capacity to synthesize asparagine by the action of L-asparagine synthetase. Some tumor cells, particularly those of T-cell lineage, lack asparagine synthesis capability and require exogenous asparagine to proliferate. As a result, depletion of circulating pools of asparagine by L-asparaginase results in inhibition of protein synthesis and ultimately cell death.

Side effects: In contrast to other chemotherapeutic drugs, asparaginase has minmal effects on bone marrow, oral and GI mucosa, or hair follicle. However, it carries the risk of coagulopathy. Hepatotoxicity is clinically evident in $10-20 \%$ of patients (increased $\mathrm{P} / \mathrm{T}$ ) and $50 \%$ have biochemical evidence of liver dysfunction.

\subsubsection{Random synthetics}

Examples of synthetic chemotherapeutic drugs are: Cisplatin, hydroxyurea, procarbazine, and mitotane. 
Cisplatin: An inorganic platinum-containing complex (a heavy metal) that enters cells by diffusion and disrupts the DNA helix. Its action is to cause DNA breaks and cross- link complimentary DNA strands that prevent replication. Renal toxicity is prominent and can lead to renal failure. Myelosuppression is also seen, along with ototoxicity (manifested by tinnitus), nausea, vomiting and peripheral sensory neuropathies.

Hydroxyurea: Acts on the enzyme ribonucleosidediphosphatereductase to interfere with the synthesis of DNA. Myelosuppression, nausea and vomiting are the major side effects.

Procarbazine: Inhibits DNA synthesis. Myelosuppression, nausea and vomiting are the major side effects. Sedative effects and depression are prominent. This drug is a weak MAO inhibitor so tricyclic anidepressant should be used with caution. Synergism occurs with barbiturates, narcotics, phenothiazines and sedatives.

\subsubsection{Hormones}

Hormones - corticosteroids, progestin, antiestrogens and antiandrogens - slow the growth of some cancers that depend on hormones.

Corticosteroids: Possess lympholytic effects and suppress mitosis in lymphocytes. They are used to treat acute lymphoma in children (not adults) and malignant lymphoma.

Progestins: Used for endometrial carcinoma because it slows the overstimulation of the endometrium which cause the neoplastic changes.

Estrogens and Androgens: Malignant changes in the breast and prostate often depend on hormones for their continued growth. For example, prostatic cancer is stimulated by androgens, so giving estrogen (diethylstilbestrol) will slow the growth of the tumor cells. Estrogens and androgens are valuable in the treatment of advanced breast cancer. Malignant tissues that are responsive to estrogens contain receptors for that hormone, whereas malignant tumors lacking these receptors are unlikely to respond hormonal manipulation. Hypercalcemia is often associated with androgen or estrogen therapy.

Antiestrogens: Tamoxifen binds to estrogen receptors and inhibits continued growth of estrogen-dependet tumors. It is used for palliative treatment of advanced cancer of the breast in postmenopausal females. Side effects are hot flashes, nausea and vomiting.

Antiandrogens: Flutamide is a nonsteroidal antiandrogenic drug used for prostate cancer. It prevents androgen binding to androgen receptors. Side effects are skeletal muscle weakness, osteoporosis and methemaglobinemia (at levels $>35 \%$ pulse oximetry readings will approach $85 \%$ )

\section{Oral and maxillofacial surgery considerations}

The bone marrow suppression caused by the chemotherapeutic agents will pose the greatest concerns to the oral and maxillofacial surgeon. Bone marrow suppression is a major side effect 
of nearly all of the widely used agents. It manifests as neutropenia, anemia and thrombocytopenia. The decreases in WBCs and platelets will be the major issues that the surgeon will need to manage.

Myelosuppression: Caused by nearly all of the chemotherapeutic agents, is reversible and should be close to normal within $6-8$ weeks after the drugs have been stopped. The surgeon should therefore allow about 2 months after chemotherapy for bone marrow to regrow. Neutropenia and thrombocytopenia will be the major concerns to the surgeon.

Neutropenia: An absolute neutrophil count (ANC) of less than $1500 / \mathrm{mm}^{3}$ The risk for infection is related to the severity and duration of the neutropenia

Categorization of neutropenia:

1,000 - 1,500 mild

$500-1000$ moderate

$<500$ severe

Patients with mild neutropenia do not require prophylactic antibiotic for routine oral surgery. The authors believe that patients with moderate neutropenia should be given prophylactic antibiotic for invasive procedures such as tooth extraction, followed by a 7 day course of antimicrobials to prevent secondary infection. Severe neutropenia cases must be given prophylactic antibiotic for any oral surgical procedure. Ciprofloxacin plus amoxicillin are recommended for adult patients who are at low risk for complications. Patients who have higher risk should receive vancomycin. This should also be followed by a 7 day course of antimicrobials to prevent secondary infection. The antibiotic should cover the normal oral flora.

Thrombocytopenia: Chemotherapy induced thrombocytopenia typically occurs $6-10$ days after administration of the drug. The risk of for excessive bleeding with invasive procedures occur at counts below $50,000 / \mathrm{mm}^{3}$ Platelet transfusion is the primary method of managing thrombocytopenia. The usual therapeutic dose for transfusion is one platelet concentrate (1 unit) per $10 \mathrm{~kg}$ of body weight. It is expected that one unit will increase the platelet count 5000 $-7000 / \mathrm{mm}^{3}$ Coagulation defects, not caused by thrombocytopenia, may be caused by mechlorethamine, mithramycin and L-asparaginase. Patients who have had these drugs should be screened by a coagulation profile and abnormalities corrected appropriately. Other noxious side effects can be managed palliatively.

\section{Bisphosphonate related osteonecrosis of the jaw (BRONJ)}

In the modern day medicine bisphosphonates are used for management of many conditions such as osteoporosis, Paget's disease, breast cancer, prostate cancer and multiple myeloma. [40]Bisphosphonate related osteonecrosis of the jaw is a pathologic condition resulting in a non-healing, necrotic sequestrate of bone in patients on past or current bisphosphonate 
therapy. The natural progression of the disease is probably similar with many patients (dental extraction in a patient with poor oral hygiene who has been on bisphosphonate therapy for a long period of time). The extraction socket does not heal, or heals but becomes covered with ulcerated overlying epithelium. Multiple exposed sites of painful bony spicules are present with occasional purulent exudate. The development of BRONJ appears to depend on the route and dose of administration of the drug as well as several other risks factors. New clinical treatments are however, being constantly discovered and it is likely that the uses of bisphosphonates will only increase in the future.

The mode of action of bisphosphonates revolves around the drug's intricate interaction with osteoclasts. [41] Once bisphosphonates are circulating in the bloodstream, they are taken up by osteoclasts, which subsequently undergo physical changes and lose their ruffled borders. [42] These structural changes in the osteoclasts alone appear to be sufficient to inhibit their bone resorptive activity. Bisphosphonates also, however, appear to directly cause apoptosis of osteoclasts and hence decrease overall number of available and viable cells. [43] Lastly bisphosphonates also inhibit the important osteoclast-osteoblast interaction, disrupting the important resorption and new deposition pattern. [44] Figure 3 summarizes relative risks of developing BRONJ.

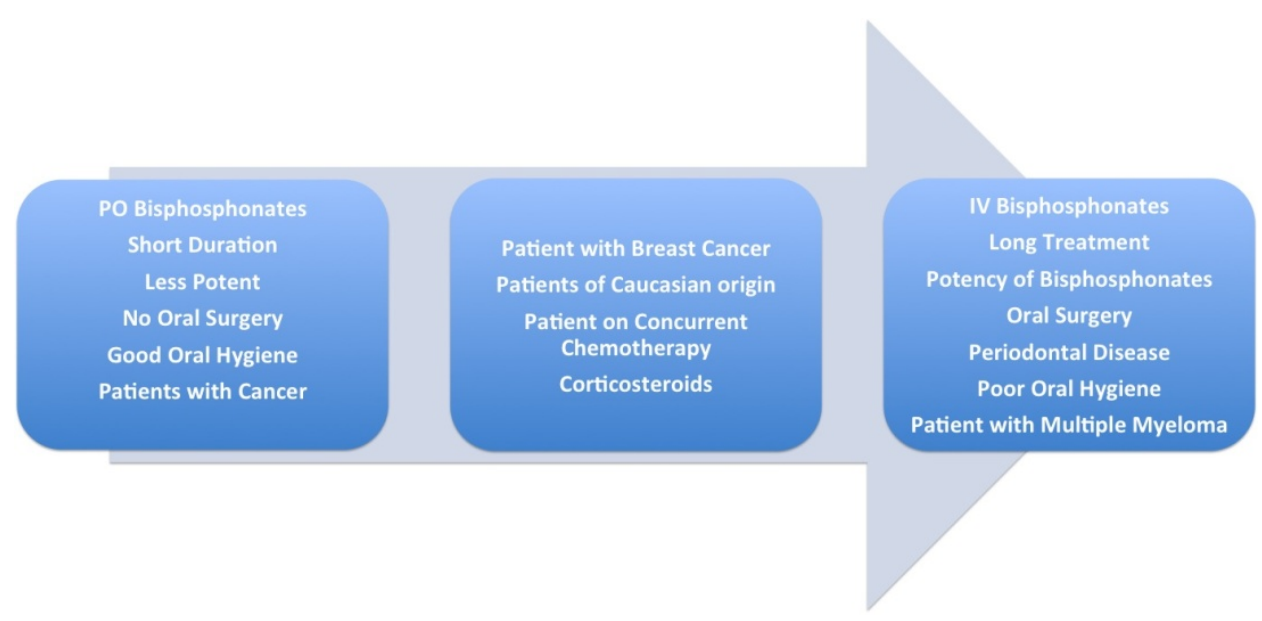

Figure 3. Risks factors for BRONJ. Modified from Bisphosphonate-related osteonecrosis of the jaws. In: Davies JEA, ed. Oral Complications of Cancer and Its Management[49]

Route of administration as well as duration appears to be the most important risks factors for developing BRONJ. [45] Most of the literature reports that there are usually triggering events (i.e. dental extractions, soft tissue trauma) before the disease makes itself visible, others suggest that disease is present long before clinical signs and symptoms become noticeable. [46, 47]Regardless of the etiology, once the disease entity is suspected appropriate staging and management options should be considered. Many clinical associations as well as American Association of Oral and Maxillofacial Surgeons have adopted 
concise definitions to facilitate staging and management of the disease. [48] Table 4 summarizes these diagnostic considerations.

\section{AAOMS BRONJ Diagnostic Criteria}

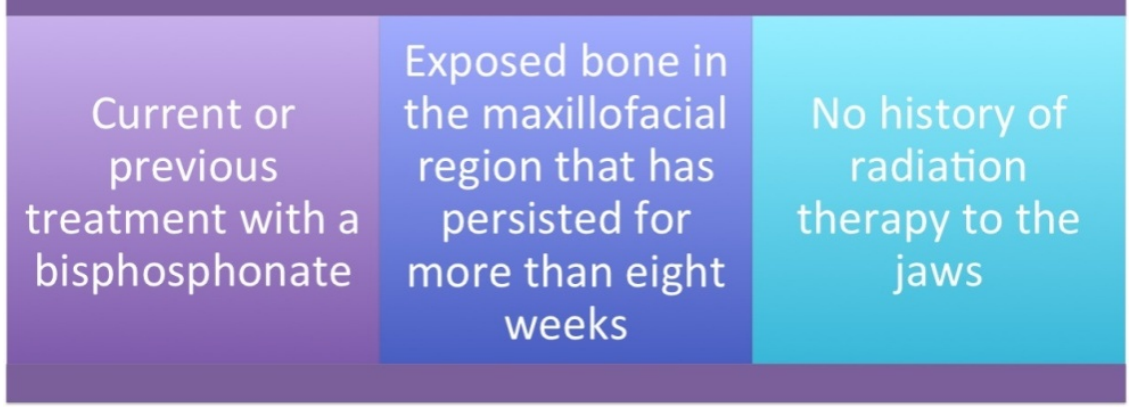

Table 4. BRONJ diagnostic criteria. Modified from BRONJ Diagnosis adopted from American Association of Oral and Maxillofacial Surgeons position paper on bisphosphonate-related osteonecrosis of the jaw. - 2009 update. Aust Endod J 2009;35[3]:119-130.

The treatment objectives for patients with an established diagnosis of BRONJ are to eliminate pain, control infection of the soft and hard tissue, and minimize the progression or occurrence of bone necrosis (see Table 5).[48]

\begin{tabular}{|c|c|}
\hline \multicolumn{2}{|c|}{ BRONJ } \\
\hline Stage & Management \\
\hline $\begin{array}{l}\text { At risk category: No apparent necrotic bone in patients who have been } \\
\text { treated with either oral or IV bisphosphonates }\end{array}$ & No treatment indicated. Patient education \\
\hline $\begin{array}{l}\text { Stage 0: No clinical evidence of necrotic bone, but non-specific clinical } \\
\text { findings and symptoms }\end{array}$ & Systemic management, including the use of pain medication and antibiotics \\
\hline $\begin{array}{l}\text { Stage 1: Exposed and necrotic bone in patients who are asymptomatic and } \\
\text { have no evidence of infection }\end{array}$ & $\begin{array}{l}\text { Antibacterial mouth rinse. Clinical follow-up on a quarterly basis. Patient } \\
\text { education and review of indications for continued bisphosphonate therapy }\end{array}$ \\
\hline $\begin{array}{l}\text { Stage 2: Exposed and necrotic bone associated with infection as evidenced } \\
\text { by pain and erythema in the region of the exposed bone with or without } \\
\text { purulent drainage }\end{array}$ & $\begin{array}{l}\text { Symptomatic treatment with oral antibiotics Oral antibacterial mouth rinse. } \\
\text { Pain control. Superficial debridement to relieve soft tissue irritation }\end{array}$ \\
\hline $\begin{array}{l}\text { Stage 3: Exposed and necrotic bone in patients with pain, infection and } \\
\text { one or more of the following: exposed and necrotic bone extending } \\
\text { beyond the region of alveolar bone (i.e. inferior border and ramus in the } \\
\text { mandible, maxillary sinus and zygoma in the maxilla) resulting in pathologic } \\
\text { fracture, extra-oral fistula, oral antral/oral nasal communication, or } \\
\text { osteolysis extending to the inferior border of the mandible of sinus floor }\end{array}$ & $\begin{array}{l}\text { Antibacterial mouth rinse Antibiotic therapy and pain control Surgical } \\
\text { debridement/resection for longer term palliation of infection and pain }\end{array}$ \\
\hline
\end{tabular}

Table 5. BRONJ Management Recommendations. Modified from BRONJ Diagnosis adopted from American Association of Oral and Maxillofacial Surgeons position paper on bisphosphonate-related osteonecrosis of the jaw. 2009 update. Aust Endod J 2009;35[3]:119-130. 


\section{Summary}

Treatment of oral cancer presents a challenge to not only Oral and Maxillofacial surgeons but also to auxiliary staff, oncologists and certainly patients and their family. Treatment is usually complex, multidisciplinary and very expensive. The chapter above presents an overview of types of oral cancers in the mouth and their treatment. The position of an oral and maxillofacial surgeon remains pivotal; first to perform definitive diagnosis and provide appropriate referral. This is a rather rapidly changing field in medicine and new advanced treatment modalities continue to emerge. So it is extremely important to remain current with the most up-to-date treatment options to better serve the needs of our patients.

\section{Author details}

Orett E. Ogle* and Levon Nikoyan

*Address all correspondence to: Oeogle@aol.com

Department of Oral and Maxillofacial Surgery, Woodhull Medical and Mental Center, Brooklyn, NY, USA

\section{References}

[1] Shah, J. P, \& Gil, Z. Current concepts in management of oral cancer--surgery. Oral Oncol. (2009). Epub 2008/08/05.

[2] Organization, W. H. Global data on incidence of oral cancer. In: Petersen PE, editor.: World Health Organization; (2005).

[3] Lee, N, Puri, D. R, Blanco, A. I, \& Chao, K. S. Intensity-modulated radiation therapy in head and neck cancers: an update. Head Neck. (2007). Epub 2005/12/17., 29(4), 387-400.

[4] Corvo, R. Evidence-based radiation oncology in head and neck squamous cell carcinoma. Radiother Oncol. (2007). Epub 2007/05/08., 85(1), 156-70.

[5] Clinical Radiation Oncologyrd ed. Leonard L. Gunderson JET, editor. Philadelphia: Saunders; (2012).

[6] Ang, K. K. Advances in the Treatment of Head and Neck Cancer. In: James D. Cox KKA, editor. Radiation Oncology, Treatment, Technique Rationale. 9th ed. Philadelphia, PA: Mosby, Elsevier; (2010). , 161-353. 
[7] Kate Newbold KHOverview of complications of radiotherapy. In: Andrew Davies JE, editor. Oral Complications of Cancer and its Management. Oxford: Oxford University Press; (2010). , 90-97.

[8] Levy, R. P, Fabrikant, J. I, Frankel, K. A, Phillips, M. H, \& Lyman, J. T. Charged-particle radiosurgery of the brain. Neurosurg Clin N Am. (1990). Epub 1990/10/01., 1(4), 955-90.

[9] Karzmark, C. J M. R. A Primer on theory and operation of linear accelerators in radiation therapy. 2nd ed. Madison: Medical Physics Publishing; (1996).

[10] Chang, D. T, Sandow, P. R, Morris, C. G, Hollander, R, Scarborough, L, Amdur, R. J, et al. Do pre-irradiation dental extractions reduce the risk of osteoradionecrosis of the mandible? Head Neck. (2007). Epub 2007/01/19., 29(6), 528-36.

[11] Deasy, J. O, Moiseenko, V, Marks, L, Chao, K. S, Nam, J, \& Eisbruch, A. Radiotherapy dose-volume effects on salivary gland function. Int J Radiat Oncol Biol Phys. (2010). Suppl):SEpub 2010/03/05., 58-63.

[12] Thomas Galloway MRobert J Amdur M. Management and prevention of complications of head and neck cancer during initial treatment. In: Marshall R Posner M, Bruce E Brockstein M, David M Brizel M, Daniel G Deschler M, FACS, editors.: UpToDate; 07/03/(2012).

[13] Koga, D. H, Salvajoli, J. V, \& Alves, F. A. Dental extractions and radiotherapy in head and neck oncology: review of the literature. Oral Dis. (2008). Epub 2008/01/05., 14(1), $40-4$.

[14] Murray, C. G, Daly, T. E, \& Zimmerman, S. O. The relationship between dental disease and radiation necrosis of the mandible. Oral Surg Oral Med Oral Pathol. (1980). Epub 1980/02/01., 49(2), 99-104.

[15] Thorn, J. J, Hansen, H. S, Specht, L, \& Bastholt, L. Osteoradionecrosis of the jaws: clinical characteristics and relation to the field of irradiation. J Oral Maxillofac Surg. (2000). discussion 93-5. Epub 2000/10/06., 58(10), 1088-93.

[16] Marx, R. E. A new concept in the treatment of osteoradionecrosis. J Oral Maxillofac Surg. (1983). Epub 1983/06/01., 41(6), 351-7.

[17] Sciubba, J. J, \& Goldenberg, D. Oral complications of radiotherapy. Lancet Oncol. (2006). Epub 2006/02/04., 7(2), 175-83.

[18] Tong, A. C, Leung, A. C, Cheng, J. C, \& Sham, J. Incidence of complicated healing and osteoradionecrosis following tooth extraction in patients receiving radiotherapy for treatment of nasopharyngeal carcinoma. Aust Dent J. (1999). Epub 1999/12/11., 44(3), 187-94.

[19] Assael, L. A. New foundations in understanding osteonecrosis of the jaws. J Oral Maxillofac Surg. (2004). Epub 2004/02/06., 62(2), 125-6. 
[20] Al-nawas, B, Duschner, H, \& Grotz, K. A. Early cellular alterations in bone after radiation therapy and its relation to osteoradionecrosis. J Oral Maxillofac Surg. (2004). Epub 2004/07/28.

[21] Costantino, P. D, Friedman, C. D, \& Steinberg, M. J. Irradiated bone and its management. Otolaryngol Clin North Am. (1995). Epub 1995/10/01., 28(5), 1021-38.

[22] Beumer, J. rd, Curtis T, Harrison RE. Radiation therapy of the oral cavity: sequelae and management, part 1. Head Neck Surg. (1979). Epub 1979/03/01., 1(4), 301-12.

[23] Beumer, J. rd, Curtis T, Harrison RE. Radiation therapy of the oral cavity: sequelae and management, part 2. Head Neck Surg. (1979). Epub 1979/05/01., 1(5), 392-408.

[24] Epstein, J, Van Der Meij, E, Mckenzie, M, Wong, F, Lepawsky, M, \& Stevensonmoore, P. Postradiation osteonecrosis of the mandible: a long-term follow-up study. Oral Surg Oral Med Oral Pathol Oral Radiol Endod. (1997). Epub 1997/06/01., 83(6), 657-62.

[25] Kampen, W. U, Brenner, W, Terheyden, H, Bohuslavizki, K. H, \& Henze, E. Decisive diagnosis of infected mandibular osteoradionecrosis with a Tc-99m-labelled antigranulocyte Fab'-fragment. Nuklearmedizin. (1999). Epub 1999/12/22., 38(7), 309-11.

[26] Mansberg, V. J, \& Frater, C. J. Development of osteoradionecrosis demonstrated on bone scintigraphy. Clin Nucl Med. (2003). Epub 2003/06/24., 28(7), 587-8.

[27] Goldwaser, B. R, Chuang, S. K, Kaban, L. B, \& August, M. Risk factor assessment for the development of osteoradionecrosis. J Oral Maxillofac Surg. (2007). Epub 2007/10/24., 65(11), 2311-6.

[28] Friedman, R. B. Osteoradionecrosis: causes and prevention. NCI Monogr. (1990). Epub 1990/01/01., 1990(9), 145-9.

[29] Epstein, J. B, Rea, G, Wong, F. L, Spinelli, J, \& Stevenson-moore, P. Osteonecrosis: study of the relationship of dental extractions in patients receiving radiotherapy. Head Neck Surg. (1987). Epub 1987/09/01., 10(1), 48-54.

[30] Jansma, J, Vissink, A, Spijkervet, F. K, Roodenburg, J. L, Panders, A. K, Vermey, A, et al. Protocol for the prevention and treatment of oral sequelae resulting from head and neck radiation therapy. Cancer. (1992). Epub 1992/10/15., 70(8), 2171-80.

[31] Pitak-arnnop, P, Sader, R, Dhanuthai, K, Masaratana, P, Bertolus, C, Chaine, A, et al. Management of osteoradionecrosis of the jaws: an analysis of evidence. Eur J Surg Oncol. (2008). Epub 2008/05/06., 34(10), 1123-34.

[32] Bennett, M. H, Feldmeier, J, Hampson, N, Smee, R, \& Milross, C. Hyperbaric oxygen therapy for late radiation tissue injury. Cochrane Database Syst Rev. (2012). CD005005. Epub 2012/05/18.

[33] What is HBO Therapy [database on the Internet)oxynet. (2003). cited 08/01/2012). Available from: http://www.oxynet.org/HBOInfo.htm. 
[34] Tompach, P. C, Lew, D, \& Stoll, J. L. Cell response to hyperbaric oxygen treatment. Int J Oral Maxillofac Surg. (1997). Epub 1997/04/01., 26(2), 82-6.

[35] Tuncay, O. C, Ho, D, \& Barker, M. K. Oxygen tension regulates osteoblast function. Am J Orthod Dentofacial Orthop. (1994). Epub 1994/05/01., 105(5), 457-63.

[36] Schwartz, H. C, \& Kagan, A. R. Osteoradionecrosis of the mandible: scientific basis for clinical staging. Am J Clin Oncol. (2002). Epub 2002/04/11., 25(2), 168-71.

[37] Jacobson, A. S, Buchbinder, D, Hu, K, \& Urken, M. L. Paradigm shifts in the management of osteoradionecrosis of the mandible. Oral Oncol. (2010). Epub 2010/09/17., 46(11), 795-801.

[38] Oh, H. K, Chambers, M. S, Garden, A. S, Wong, P. F, \& Martin, J. W. Risk of osteoradionecrosis after extraction of impacted third molars in irradiated head and neck cancer patients. J Oral Maxillofac Surg. (2004). Epub 2004/02/06., 62(2), 139-44.

[39] Ueda, M, Kaneda, T, \& Takahashi, H. Effect of hyperbaric oxygen therapy on osseointegration of titanium implants in irradiated bone: a preliminary report. Int J Oral Maxillofac Implants. (1993). Epub 1993/01/01., 8(1), 41-4.

[40] Drake, M. T, Clarke, B. L, \& Khosla, S. Bisphosphonates: mechanism of action and role in clinical practice. Mayo Clin Proc. (2008). Epub 2008/09/09., 83(9), 1032-45.

[41] Santini, D. Vespasiani Gentilucci U, Vincenzi B, Picardi A, Vasaturo F, La Cesa A, et al. The antineoplastic role of bisphosphonates: from basic research to clinical evidence. Ann Oncol. (2003). Epub 2003/09/25., 14(10), 1468-76.

[42] Murakami, H, Takahashi, N, Sasaki, T, Udagawa, N, Tanaka, S, Nakamura, I, et al. A possible mechanism of the specific action of bisphosphonates on osteoclasts: tiludronate preferentially affects polarized osteoclasts having ruffled borders. Bone. (1995). Epub 1995/08/01., 17(2), 137-44.

[43] Ito, M, Amizuka, N, Nakajima, T, \& Ozawa, H. Ultrastructural and cytochemical studies on cell death of osteoclasts induced by bisphosphonate treatment. Bone. (1999). Epub 1999/10/08., 25(4), 447-52.

[44] Hughes, D. E. MacDonald BR, Russell RG, Gowen M. Inhibition of osteoclast-like cell formation by bisphosphonates in long-term cultures of human bone marrow. J Clin Invest. (1989). Epub 1989/06/01., 83(6), 1930-5.

[45] Bamias, A, Kastritis, E, Bamia, C, Moulopoulos, L. A, Melakopoulos, I, Bozas, G, et al. Osteonecrosis of the jaw in cancer after treatment with bisphosphonates: incidence and risk factors. J Clin Oncol. (2005). Epub 2005/11/30., 23(34), 8580-7.

[46] Ruggiero, S. L, Mehrotra, B, Rosenberg, T. J, \& Engroff, S. L. Osteonecrosis of the jaws associated with the use of bisphosphonates: a review of 63 cases. J Oral Maxillofac Surg. (2004). Epub 2004/05/04., 62(5), 527-34.

[47] Migliorati, C. A, Schubert, M. M, Peterson, D. E, \& Seneda, L. M. Bisphosphonateassociated osteonecrosis of mandibular and maxillary bone: an emerging oral com- 
plication of supportive cancer therapy. Cancer. (2005). Epub 2005/06/02., 104(1), 83-93.

[48] Ruggiero, S. L, Dodson, T. B, Assael, L. A, Landesberg, R, Marx, R. E, \& Mehrotra, B. American Association of Oral and Maxillofacial Surgeons position paper on bisphosphonate-related osteonecrosis of the jaw- 2009 update. Aust Endod J. (2009). Epub 2009/12/08., 35(3), 119-30.

[49] James Sciubba JEBisphosphonate-related osteonecrosis of the jaws. In: Davies JEA, editor. Oral Complications of Cancer and Its Management. Oxford: Oxford University Press; (2010). , 151-163. 